\title{
WAVELET-BASED ANALYSIS OF CEREBROVASCULAR DYNAMICS IN NEWBORN RATS WITH INTRACRANIAL HEMORRHAGES
}

\author{
ALEXEY N. PAVLOV*, ALEXEY I. NAZIMOV, OLGA N. PAVLOVA, \\ VLADISLAV V. LYCHAGOV and VALERY V. TUCHIN \\ Department of Physics, Saratov State University \\ 83 Astrakhanskaya str., Saratov 410012, Russia \\ *pavlov.lesha@gmail.com \\ OLGA A. BIBIKOVA, SERGEY S. SINDEEV and \\ OXANA V. SEMYACHKINA-GLUSHKOVSKAYA ${ }^{\dagger}$ \\ Department of Biology, Saratov State University \\ 83 Astrakhanskaya str., Saratov 410012, Russia \\ †glushkovskaya@mail.ru
}

Received 13 May 2013

Accepted 12 September 2013

Published 7 November 2013

\begin{abstract}
Intracranial hemorrhage (IH) is a major problem of neonatal intensive care. The incidence of $\mathrm{IH}$ is typically asymptomatic and cannot be effectively detected by standard diagnostic methods. The mechanisms underlying IH are unknown but there is evidence that stress-induced disorders in adrenergic regulation of cerebral venous blood flow (CVBF) are among the main reasons. Quantitative and qualitative assessment of CVBF could significantly advance understanding of the nature of IH in newborns. In this work, we analyze variations of CVBF in newborn rats with an experimental model of stress-induced IH and adrenaline injection. Our analysis is based on the Doppler optical coherence tomography (DOCT) and a proposed adaptive wavelet-based approach that provides sensitive markers of abnormal reactions of the sagittal vein to external factors. The obtained results demonstrate that the incidence of $\mathrm{IH}$ in newborn rats is accompanied by a suppression of CVBF with the development of venous insufficiency and areactivity to adrenaline. We introduce a numerical measure $\theta$, quantifying reactions of CVBF and show that the values $\theta<1.23$ estimated in the low-frequency (LF) spectral range corresponding to the sympathicus indicate abnormal reactions associated with the development of IH. We conclude that the revealed
\end{abstract}

${ }^{*}$ Corresponding author.

This is an Open Access article published by World Scientific Publishing Company. It is distributed under the terms of the Creative Commons Attribution 3.0 (CC-BY) License. Further distribution of this work is permitted, provided the original work is properly cited. 


\section{A. N. Pavlov et al.}

areactivity of the cerebral veins to adrenaline represents a possible mechanism responsible for pathological changes in CVBF.

Keywords: Brain hemorrhages; optical coherence tomography; cerebrovascular dynamics; wavelet analysis; stress.

\section{Introduction}

Intracranial hemorrhage (IH) is a major reason of neonatal morbidity and mortality that was revealed in about $50 \%$ premature infants who were born at 24-26 weeks gestation, in 15-30\% full-term babies with deep hypoxia and with birth trauma. ${ }^{1-3}$ The incidence of IH is typically asymptomatic and cannot be effectively detected by standard diagnostic methods. Mechanisms responsible for the development of IH in neonates are poorly understood, but there is a strong evidence that impairments of cerebral venous blood flow (CVBF) serve as the main reason. ${ }^{4,5}$ The sympathetic nervous system plays a key role in the regulation of cerebral circulation from early stages of the development ${ }^{6,7}$ as well as in the protective mechanism for the brain, limiting cerebral perfusion and microcirculatory pressure after $\mathrm{IH} .{ }^{8-12}$ Despite intensive investigations in this field, functional role of the sympathetic nervous system in the regulation of the cerebral hemodynamics remains disputable. ${ }^{13}$ At present, there is few information regarding age particularities of the adrenergic control of the cerebral circulation..$^{9,13}$

The IH generally occurs during the second postnatal day (about $48 \mathrm{~h}$ after birth). ${ }^{14,15}$ During this critical period, most infants demonstrate subtle signs that are easily overlooked and cannot be readily detected with the most widely used neuroimaging procedures. Furthermore, standard methods for imaging of cerebral blood vessels and for measurement of CVBF such as the magnetic resonance imaging (MRI) and the emission tomography have some limitations for newborns or they do not provide necessary information about time-varying changes of $\mathrm{CVBF}$ and mechanisms responsible for its regulation. ${ }^{16,17}$ Therefore, studies of the disease progression or responses to treatment cannot be performed using these methods. Absence of effective diagnostic technologies for early recognition of IH explains a high percentage of neonatal death and less optimistic neurologic prognosis in infants after IH. ${ }^{18-21}$ Development of noninvasive techniques to provide diagnostics of IH risk is of high importance.
For this purpose, we consider here an approach based on the optical coherence tomography (OCT).

A promising trend in modern biomedical optics is associated with the correlation analysis of multiple scattered light fields generated by interactions of coherent or partially coherent light with biological tissues. OCT is one from the methods implementing this principle that has found various applications in medicine. ${ }^{2-24}$ OCT enables noninvasive real-time analyses of blood supply of the brain tissue by monitoring the CVBF parameters in the surface vessels. As the main tool for characterizing a timevarying dynamics of cerebral vessels we use a wavelet-based technique. Advantages of wavelets over the classical spectral methods are widely discussed. $^{25,26}$ Thus, the multiresolution analysis based on the discrete wavelet transform can propose informative measures of heart failure. ${ }^{27}$ The wavelet-based multifractal formalism reveals early stages of transformation of normal physiological processes in the cardiovascular dynamics into the pathological processes. ${ }^{28}$ This technique is also effective for quantifying responses of vessels dynamics to varying environmental conditions. ${ }^{29} \mathrm{In}$ this paper, we propose an adaptive wavelet-based approach aiming to reveal instantaneous characteristics of blood flow dynamics from OCT data and to diagnose abnormalities in the dynamics of brain vessels.

\section{Materials and Methods}

\subsection{Experimental procedures}

Experiments were performed in 12 mongrel newborn male rats (2-3 days after birth) in accordance with the Guide for the Care and Use of Laboratory Animals published by the US National Institutes of Health (NIH Publication No. 85-23, revised 1996); protocols of experimental study were approved by the Institutional Review Boards of the Saratov State University. The animals were housed at $25 \pm 2{ }^{\circ} \mathrm{C}$, $55 \%$ humidity and 12:12 h light-dark cycle. 
Monitoring of CVBF in the superior sagittal vein was performed in anesthetized rats (thiopental anesthesia, $40 \mathrm{mg} / 1000 \mathrm{~g}$ ) with the fixed head after the scalp incision. Analysis of CVBF was carried out under normal condition and the next day after the stress. To study the effects of adrenergic influences on CVBF we analyzed a sensitivity of sagittal vein to adrenaline $(10 \mathrm{~g} / \mathrm{kg}$, Sigma, iv) in healthy newborn rats $(n=7)$ and in stressed rats with $\mathrm{IH}$ $(n=5)$.

To induce IH, the newborn rats underwent intermittent infrasound $(10 \mathrm{~Hz}, 120 \mathrm{~dB}): 10 \mathrm{~s}$ - the sound, then $60 \mathrm{~s}$ - the interruption and this cycle was repeated for $2 \mathrm{~h}$. This procedure was performed in the Plexiglas chamber (the volume $-2000 \mathrm{sm}^{3}$ ) absorbing and amplifying deleterious effects of infrasound on the rats. The incidence of IH in newborn rats was detected using T1.5-weighted MRI of the brain (Siemens MAGNETON 1.5T, Germany). The hyperintense signal of the T1.5-weighted MRI in newborn rats was an indicator of superficial multiple IH in the brain. In our previous study ${ }^{30}$ we obtained that infrasound-induced IH in newborn and adult rats is accompanied by engorgement of sagittal sinus with the fall of velocity of blood flow reflecting suppression of venous blood outflow and development of venous insufficiency.

Analysis of CVBF was performed using the Doppler optical coherence tomography (DOCT) that visualizes the internal structure of an object by optical correlation analysis of reflected light. This light includes a number of elementary waves reflected from the object's surface and from any optical inhomogeneities and boundaries between layers within the object. Depending on the depth of these inhomogeneities/boundaries, the reflected elementary waves have different time delays associated with the varying length of the optical path. To quantify this time delay, the reference wave is applied. DOCT is a specialized algorithm for processing OCT data that is able to recover information about the instantaneous frequency of the interference signal at each point of the OCT image. This frequency is proportional to the velocity of inhomogeneity, from that the object wave is reflected. We used in this study a commercially available swept source DOCT system (OCS1300SS; Thorlabs, Inc, USA). Each recording contained 50 OCT images for baseline and for the state after the administration of adrenaline acquired with the time step $\Delta \tau=0.14 \mathrm{~s}$.

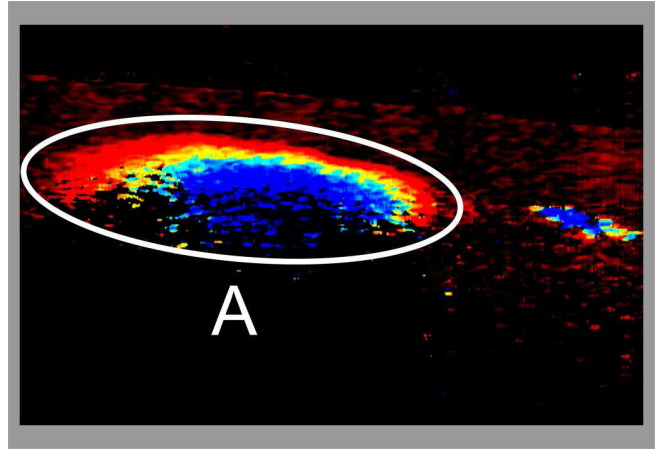

Fig. 1. Doppler OCT image of the sagittal vein in a rat.

\subsection{Data processing}

Figure 1 illustrates a fragment of the Doppler OCT image where we shall concentrate on the dynamics inside the sagittal vein that is marked by the ellipse "A". Within this ellipse, the color is associated with the velocity of scattering particles. Aiming to quantify the velocity, a segment with the height $\Delta X_{p}$ and the width $\Delta Y_{p}$ is selected. Averaged intensities $i$ for red $\left(I_{r}\right)$, green $\left(I_{g}\right)$ and blue $\left(I_{b}\right)$ colors (here, we use averaging over a square of $5 \times 5$ pixels $^{2}$ ) are estimated according to the following expression:

$$
i_{c}=\frac{1}{\left\|\Delta X_{p}\right\|\left\|\Delta Y_{p}\right\|} \int_{\Delta Y_{p}} \int_{\Delta X_{p}} I_{c}(x, y) d x d y
$$

where $c=(r, g, b)$.

Equation (1) is written for a static case (see Fig. 1). In order to provide a more detailed analysis of vessels dynamics, time dependencies $i_{c}(t)$ need to be studied. Consider a series of images $i(k \Delta \tau)$ acquired with a fixed time interval $\Delta \tau$ and construct a time dependence $s(t)$ based on Eq. (1):

$$
s(t)=i_{r}(t)-i_{b}(t), \quad t=k \Delta \tau, k \in Z .
$$

Analysis of $s(t)$ provides a way to reveal the responses of blood vessels to external factors (e.g., to adrenaline). OCT recordings of rat's sagittal veins are compared for two states: the basic state and the state after the administration of adrenaline. To perform such a comparison, an appropriate adjustment of the used technique for data processing seems to be advisable. Let us divide an original process $s$ into two parts, $s_{0}$ and $s_{1}$ associated with two mentioned states, respectively. Influence of the sampling step $\Delta \tau$ is reduced by interpolation (e.g., with cubic splines) that may be especially important when performing analysis of OCT recordings 


\section{A. N. Pavlov et al.}

with varying time intervals between samplings. As a result, the step $\Delta \tau$ is changed by a smaller value $\Delta t<\Delta \tau$, and the interpolated signals $S_{0}(t)$ and $S_{1}(t)$ contain an increased amount of data points. Next step of data preprocessing consists in the filtering of the given signals with a low-pass filter to remove high-frequency artifacts.

As a simple option, moving averages of $S_{0}(t)$ and $S_{1}(t)$ can be applied to get smoother processes $F_{0}(t)$ and $F_{1}(t)$ (see Fig. 2). Analysis of the filtered signals $F_{0}(t)$ and $F_{1}(t)$ in the frequency domain was provided using two standard approaches: Fourier- and wavelet transforms. Fourier transform is the fundamental algorithm used to compute the spectral density $S_{F}(f)$ of a process $F(t)$.

$$
\begin{aligned}
S_{F}(f) & =\left|\int_{T} F(t) \mathrm{e}^{-2 \pi f t} d t\right|^{2} \\
& \simeq\left|\sum_{j=0}^{N_{F}-1} F(j \Delta t) \mathrm{e}^{-\frac{2 \pi}{N_{F}} j k} \Delta t\right|^{2} .
\end{aligned}
$$

Here we consider the signal $F(t)$ consisting of $N_{F}$ samples $\left(N_{F} \Delta t=T\right)$. The values of $S_{F}$ are sampled with the step $\Delta f$. In order to get statistical characteristics of spectral properties, averaging over

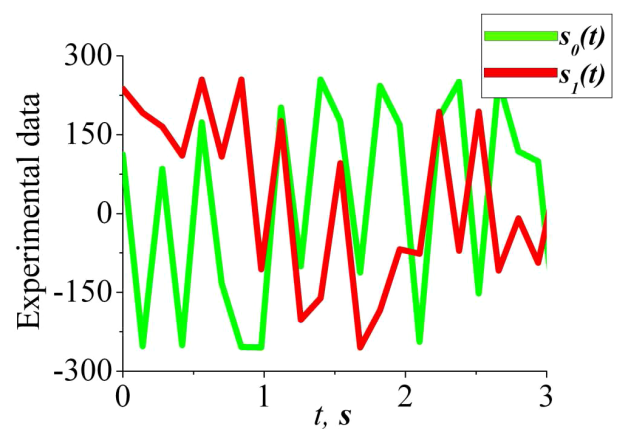

(a)

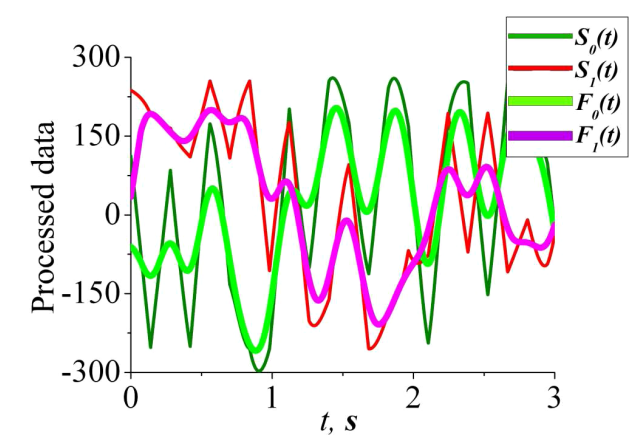

(b)

Fig. 2. Original signals (a) and results of data preprocessing (b).
$P_{F}$ segments of the duration $M_{F}\left(P_{F} M_{F}=N_{F}\right)$ is provided

$$
S_{F}(k \Delta f)=\frac{1}{P_{F}} \sum_{n=1}^{P_{F}} S_{F}^{n}(k \Delta f),
$$

$n=1,2, \ldots, P_{F}, \Delta f=\left(M_{F} \Delta t\right)^{-1}$.

Another spectral technique is based on the continuous wavelet transform of the signal $F(t)$

$$
\begin{aligned}
W(\rho, q) & =\sqrt{\rho} \int_{T} F(t) \varphi(\rho(t-q)) d t \\
& \simeq \sqrt{\rho} \sum_{k=1}^{N_{F}} F(t) \varphi(\rho(t-q)) \Delta t .
\end{aligned}
$$

Here, $t=k \Delta t, q=j \Delta t, k, j \in Z, \varphi(t)$ is the wavelet basic function, $\rho$ and $q$ characterize scaling and translation of the wavelet. By changing these quantities the transform (5) offers a two-dimensional time-frequency representation of $F(t)$. Besides the averaged (global) energy density, this approach introduces the notion of a local energy $E(q)$ being useful to characterize time-varying spectral properties

$$
E(q)=\frac{1}{\rho_{\max }-\rho_{\min }} \int_{\rho_{\min }}^{\rho_{\max }}|W(\rho, q)|^{2} d \rho .
$$

Main features of the continuous wavelet transform (5) are described in Refs. 25 and 26. As $\varphi(t)$ is defined considering the Morlet function that is characterized by an additional parameter $\omega_{0}$ called as the central frequency:

$$
\varphi(t)=\pi^{-1 / 4} \exp \left(j \omega_{0} t\right) \exp \left(-\frac{t^{2}}{2}\right) .
$$

Appropriate selection of $\omega_{0}$ realizes a compromise between time and frequency resolution of the wavelet (7) by defining the number of oscillations of the soliton-like function $\varphi(t)$. A relation between the frequency of the Fourier spectrum $f$ and the parameters $\rho$ and $\omega_{0}$ is described by the following expression

$$
f=\rho \frac{\omega_{0}+\sqrt{\omega_{0}^{2}+2}}{4 \pi} .
$$

\subsection{Proposed adaptive approach}

The proposed approach is based on the optimization theory and assumes a selection of parameters of the continuous wavelet transform that provide the most distinct separation between different physiological 
states (in our case, the clearest reaction to the administration of adrenaline). The following algorithm is suggested for solving this problem:

(1) Acquiring of OCT data associated with two physiological states (before and after the administration of adrenaline);

(2) Construction of an objective function to adjust wavelet parameters using the acquired data;

(3) Optimal configuration of the wavelet transform and definition of basic criteria to quantify normal and abnormal reactions to the administration of adrenaline;

(4) Verification of the adapted algorithm in the statistical analysis of a series of experiments.

Let us discuss this approach in more detail. According to Eq. (5), a series of wavelet coefficients $W$ is obtained as the result of the continuous wavelet transform. These coefficients depend on the parameters $\rho, q$ and $\omega_{0}$. Therefore, one requires selecting these parameters in such a way that the objective function reaches its extreme value, and the wavelet coefficients $W$ become the characteristics for signal classification.

We shall consider a selection that is based on the local mean values. The signal $F(t), t=n \Delta t$, $n=1,2, \ldots, N_{F}$ is divided into fragments $F_{i}^{j}$, $i=1,2, \ldots, M_{F}, j=1,2, \ldots, P_{F}, N_{F}=M_{F} P_{F}$ (see Fig. 3). In this case, the transform (5) takes the form

$$
W_{j}(\rho, q)=\sqrt{\rho} \sum_{i} F_{i}^{j} \varphi(\rho \Delta t(i-q)) \Delta t .
$$

Mean values $A_{j}(\rho)$ associated with the parameter $\rho$ are further estimated

$$
A_{j}(\rho)=\frac{1}{M_{F}} \sum_{k=1}^{M_{F}}\left|W_{j}(\rho, k \Delta t)\right| .
$$

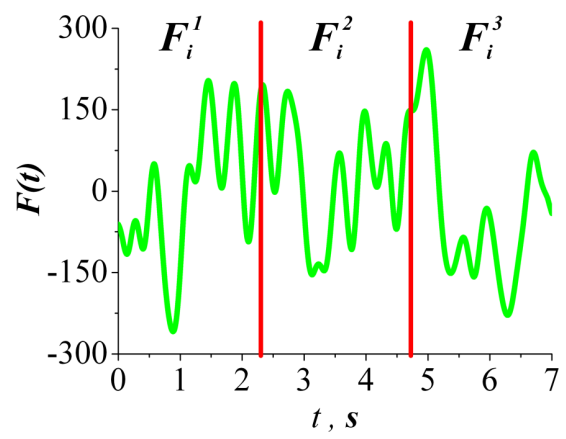

Fig. 3. Fragmentation of the analyzed signal.
Note, that these values additionally depend on the parameter $\omega_{0}$ of the Morlet function $A_{j}(\rho)=$ $A_{j}\left(\rho, \omega_{0}\right)$. Performing fragmentation of signals associated with different physiological states, two series of mean values, $A_{j}^{0}\left(\rho, \omega_{0}\right)$ and $A_{j}^{1}\left(\rho, \omega_{0}\right)$ are obtained. The objective function $R_{d}$ is constructed as follows:

$$
R_{d}\left(\rho, \omega_{0}\right)=\frac{\left\langle A_{j}^{1}\left(\rho, \omega_{0}\right)\right\rangle-\left\langle A_{j}^{0}\left(\rho, \omega_{0}\right)\right\rangle}{\sigma\left(A_{j}^{1}\left(\rho, \omega_{0}\right)\right)+\sigma\left(A_{j}^{0}\left(\rho, \omega_{0}\right)\right)},
$$

where angle brackets denote the averaging procedure, and $\sigma$ is the standard deviation. Correct selection of the parameter set corresponds to the values $\left|R_{d}\left(\rho, \omega_{0}\right)\right| \geq 1$. In the opposite case, the signals $F_{0}(t)$ and $F_{1}(t)$ have fairly small distinctions that cannot clearly be revealed based on the time-frequency analysis of OCT data.

Aiming to find extremes of the function (11), various optimization techniques can be applied including stochastic optimization based on MonteCarlo methods, ${ }^{31,32}$ gradient methods, etc. Here we use an approach of stochastic optimization that supposes a random search of parameters being optimal according to the value of the objective function (11). This approach consists of several stages:

(1) Selection of the basic wavelet (here we use the Morlet function, however, many other wavelets can also be applied).

(2) Determination of the range for parameters $\rho$ and $\omega_{0}$ that should be optimized: $\rho_{\max }=$ $1.5 / \Delta t, \rho_{\min }=1.0 / N_{F}, \omega_{\max }=\pi / \Delta t, \omega_{\min }=\pi$.

(3) Search for parameters $\rho$ and $\omega_{0}$ leading to extreme values of the objective function (11). This search is performed to achieve the indicated density of covering of the parameter plane $\left(\rho, \omega_{0}\right)$.

(4) Estimation of the maximal value of $\left|R_{d}\left(\rho, \omega_{0}\right)\right|$; this maximum corresponds to the optimal values of $\rho$ and $\omega_{0}$.

The proposed adaptive algorithm reveals the most essential spectral distinctions between the processes $F_{0}(t)$ and $F_{1}(t)$. In order to introduce diagnostics criteria based on the objective function (11), we shall use two quantities: the threshold value of $R_{d}$

$$
\theta=\frac{\left\langle\left|R_{d}\right|\right\rangle^{n}+\left\langle\left|R_{d}\right|\right\rangle^{p}}{2}
$$


and the difference coefficient

$$
\alpha=\frac{\left\langle\left|R_{d}\right|\right\rangle^{n}}{\left\langle\left|R_{d}\right|\right\rangle^{p}},
$$

where $\left\langle\left|R_{d}\right|\right\rangle^{n},\left\langle\left|R_{d}\right|\right\rangle^{p}$ are mean values of $\left|R_{d}\left(\rho, \omega_{0}\right)\right|$ for normal and pathological reactions of vessels to the administration of adrenaline.

\section{Results and Discussion}

In the first step of our study, we investigated parameters of CVBF in healthy newborn rats and in rats with $\mathrm{IH}$. The diameter of the superior sagittal vein is larger in rats with $\mathrm{IH}$ compared with healthy rats $(0.72 \pm 0.02 \mathrm{~mm}$ versus $0.33 \pm 0.03 \mathrm{~mm}, p<$ $0.05)$. Here and further, results are given as mean \pm standard error of the mean; differences between groups are evaluated by Students test with the significance level $p<0.05$. Relaxations of the sagittal vein are accompanied by a reduced velocity of CVBF that is more pronounced in rats with $\mathrm{IH}$ than in healthy animals $(3.03 \pm 0.05 \mathrm{~mm} / \mathrm{s}$ versus $6.09 \pm 0.03 \mathrm{~mm} / \mathrm{s}, p<0.05)$. These results suggest that IH in newborn rats is associated with a decreased outflow of the venous blood and with the development of venous insufficiency.

In the second step of the study, we analyzed sensitivity of the sagittal vein to adrenaline in healthy newborn rats and in rats with IH. In healthy rats, the administration of adrenaline was accompanied by a reduction of the diameter of the sagittal vein $(0.19 \pm 0.04 \mathrm{~mm}$ versus $0.33 \pm 0.03 \mathrm{~mm}, p<0.05)$ although the rate of CVBF did not demonstrate significant changes $(6.52 \pm 1.19$ versus $6.00 \pm 1.24)$. Rats with IH did not reveal changes in the diameter $(0.68 \pm 0.04 \mathrm{~mm}$ versus $0.72 \pm 0.02 \mathrm{~mm})$ and the velocity of blood flow $(2.98 \pm 0.07 \mathrm{~mm} / \mathrm{s}$ versus $3.03 \pm 0.05 \mathrm{~mm} / \mathrm{s}$ ) after the adrenaline injection. Examples of the corresponding DOCT images are shown in Fig. 4.

In the third step of the work, we studied the reactivity of the superior sagittal veins to adrenaline in healthy rats and in rats with IH based on the classical spectral analysis and the proposed adaptive technique. OCT data were analyzed in 5 rats, including 1 animal with IH. Acquired processes $s_{0}(t)$ and $s_{1}(t)$ were resampled with the time step $\Delta t=4.7 \cdot 10^{-4} \mathrm{~s}\left(N_{F}=15,000\right)$, filtered using the procedure of running averages (3 averages within a window consisting of 100 samples) and then divided

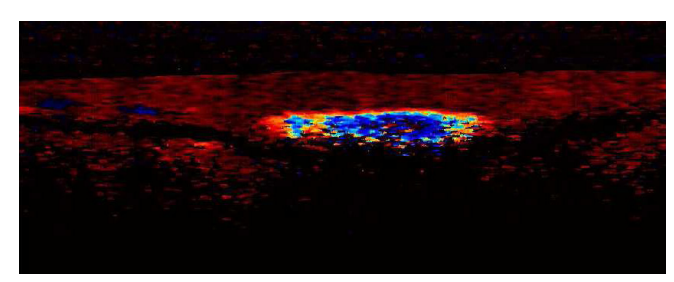

(a)

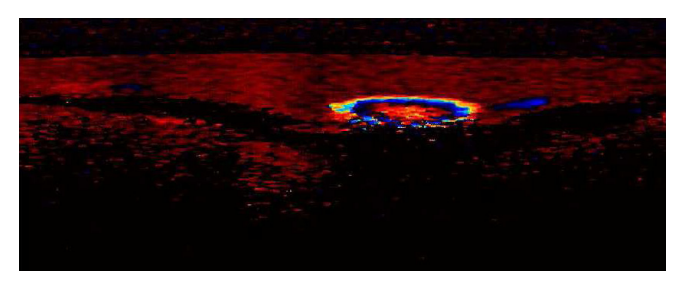

(b)

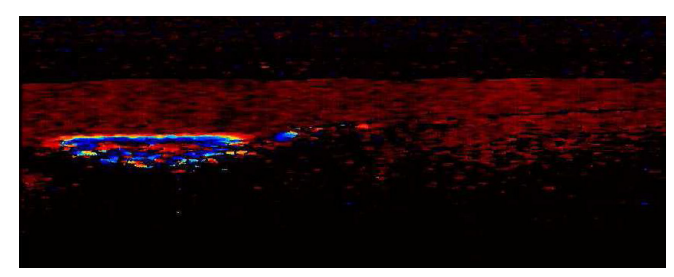

(c)

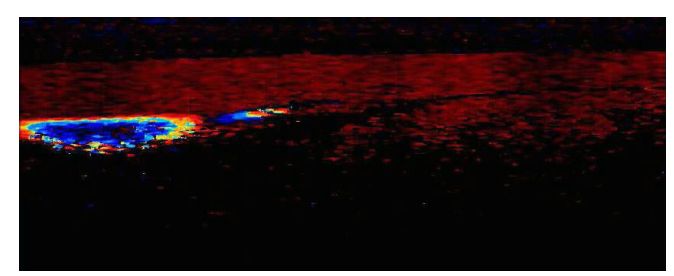

(d)

Fig. 4. Responses of the sagittal vein to adrenaline for a healthy rat (a) and (b) and a rat with $\mathrm{IH}$ (c) and (d). Baseline DOCT images are shown in (a) and (c).

into $P_{F}=3$ segments of the length $M_{F}=5000$. Spectral densities estimated with the classical Fourier analysis are given in Fig. 5. Aiming to provide a clearer physiological interpretation of the results, frequency bands associated with physiological control mechanisms were analyzed. Thus, we considered the low-frequency ( $\mathrm{LF}, 0.25-0.75 \mathrm{~Hz}$ ), the high-frequency $(\mathrm{HF}, 0.75-3.0 \mathrm{~Hz})$ and the heart rate $(\mathrm{HR}, 5.0-10.0 \mathrm{~Hz})$ dynamics. However, no obvious distinctions between the dynamics before and after the administration of adrenaline are found within all these frequency bands.

Power spectra shown in Fig. 5 do not reflect significant reactions of the sagittal vein to 


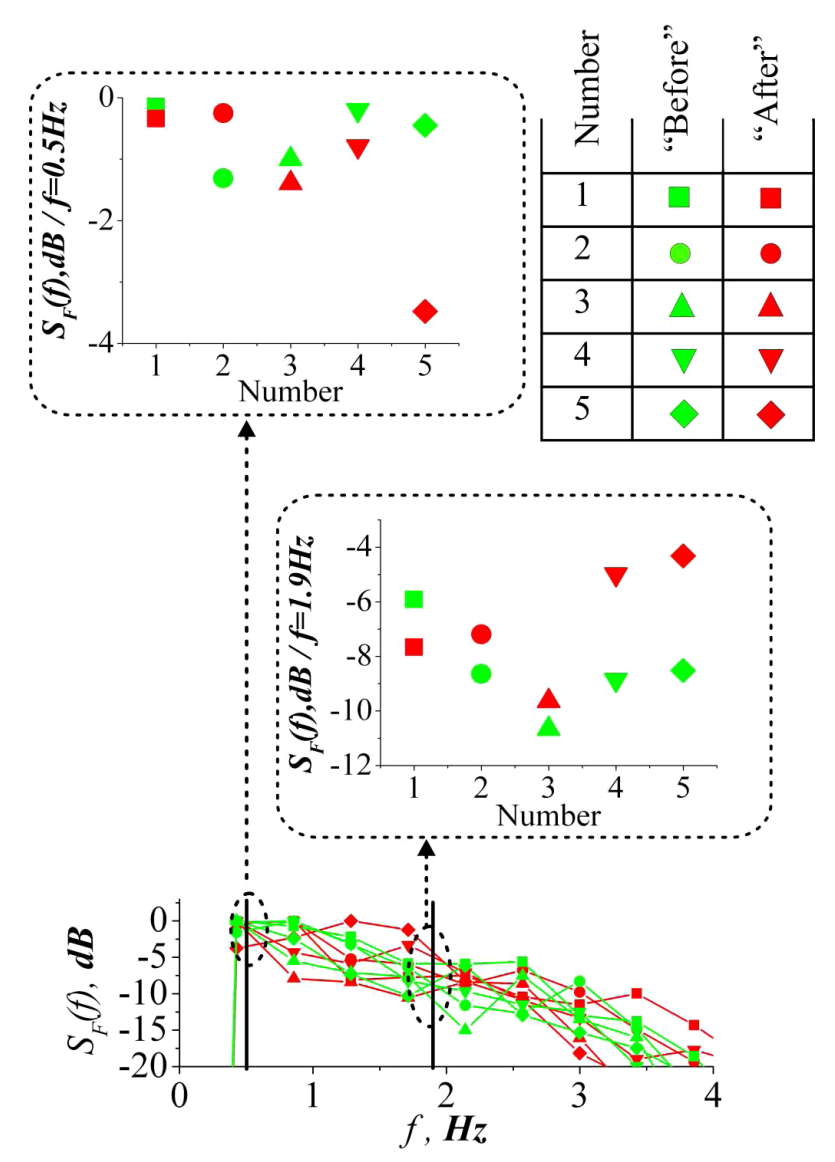

Fig. 5. Results of the classical spectral analysis. Green and red colors mark the dynamics in basic state and after the administration of adrenaline, respectively.

adrenaline. This is illustrated by inserts where spectral densities corresponding to different frequency bands and physiological states are given. As examples, we selected the frequencies 0.5 and $1.9 \mathrm{~Hz}$. The results presented in Fig. 5 demonstrate limitations of the averaged spectral characteristics for quantifying transitions between the considered physiological states and the question arises whether a localized analysis based on soliton-like functions is able to improve this situation.

Aiming to testify opportunities of the wavelet based techniques, we have applied the proposed adaptive method to the same dataset. Optimization of the objective function (11) was realized using the procedure described in Sec. 2.3. Optimal parameters of the Morlet function were selected within LF, HF and HR frequency bands. Results of this optimization are shown in Fig. 6.

Classification of distinct reactions of vessels associated with transitions between different physiological states was provided based on the values of

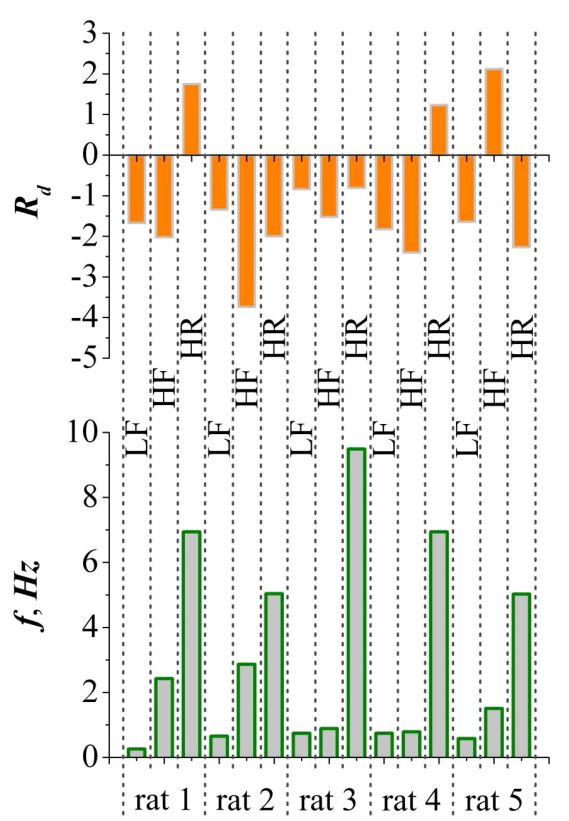

Fig. 6. Results of optimization of the objective function $R_{d}$.

$R_{d}$. This function reaches maxima within each of the analyzed frequency bands, and the corresponding frequencies can take fairly similar values. The sign of $R_{d}$ indicates whether the considered rhythmic component is more expressed after or before the administration of adrenaline. Our study has shown that the classification based on the objective function $R_{d}$ is clearer and more authentic compared with the performed analysis of energy spectra. Although the local energy $E(q)$ facilitates the revealing of distinct reactions of vessels to adrenaline, this approach is less effective compared with the $R_{d}$ analysis and only provides nonsignificant improvement of the results obtained with the classical spectral analysis (see Fig. 5). Characteristics of the analyzed test signals are given in Table 1.

Using the results shown in Table 1, the quantitative measures (12) and (13) are estimated that separate normal and abnormal reactions of the sagittal veins to adrenaline. The clearest classification of the reactions was revealed in the LF frequency area that is characterized by the quantities $\theta=1.23, \alpha=1.93$. The values $R_{d}<\theta$ are associated with pathological reactions (the case of $\mathrm{IH}$ ) and this result is in good agreement with experimental observations of fairly small changes in the diameter of the sagittal vein after the administration of adrenaline in rats with brain hemorrhages compared with stronger dilatations of the sagittal vein in normal rats (see Fig. 4). 


\section{A. N. Pavlov et al.}

Table 1. Characteristics estimated within the analyzed OCT data.

\begin{tabular}{lcrrrr}
\hline Range & Rat & \multicolumn{1}{c}{$R_{d}$} & $\rho$ & $\omega_{0}$ & $f, \mathrm{~Hz}$ \\
\hline LF & 1 & -1.67 & 1.16 & 1.05 & 0.26 \\
& 2 & -1.34 & 1.25 & 3.14 & 0.65 \\
& 3 & -0.84 & 1.41 & 3.14 & 0.74 \\
& 4 & -1.82 & 1.41 & 3.14 & 0.74 \\
& 5 & -1.64 & 1.56 & 2.09 & 0.58 \\
\hline HF & 1 & -2.02 & 6.60 & 2.09 & 2.43 \\
& 2 & -3.74 & 1.71 & 10.47 & 2.87 \\
& 3 & -1.52 & 1.29 & 4.19 & 0.89 \\
& 4 & -2.40 & 1.50 & 3.14 & 0.78 \\
& 5 & 2.12 & 2.20 & 4.19 & 1.51 \\
\hline HR & 1 & 1.75 & 1.22 & 35.60 & 6.94 \\
& 2 & -2.00 & 13.69 & 2.09 & 5.04 \\
& 3 & -0.81 & 13.85 & 4.19 & 9.49 \\
& 4 & 1.23 & 1.22 & 35.60 & 6.94 \\
& 5 & -2.27 & 3.33 & 9.42 & 5.03 \\
\hline
\end{tabular}

The considered small group (5 rats) was used at the first stage aiming to introduce sensitive diagnostic markers that could distinguish adrenalinebased reaction of the sagittal vein in normal rats and in animals with IH. The corresponding OCT data were used for appropriate adjustment of the adaptive algorithm described in Sec. 2.3. After such adjustment, OCT recordings acquired within an additional series of experiments in rats were analyzed ( 7 animals, including 3 rats with normal dynamics of brain vessels and 4 rats with $\mathrm{IH}$ ). The performed analysis confirmed the effectiveness of the quantities $\theta$ and $\alpha$ for diagnostic purposes. The value $R_{d}$ passed below the threshold value $\theta=1.23$ only for one rat related to the healthy group $\left(R_{d}=1.07\right)$. This circumstance may reflect initial stages of transformations of normal physiological processes into pathological processes.

Thus, the results of this study show a capability of wavelet-based techniques in providing sensitive markers of abnormal reactions of the sagittal vein to external factors (such as adrenaline). An essential shortcoming of the techniques that use wavelets for recognition purposes is the selection of parameters of the wavelet transform. This selection is often performed quite arbitrarily that significantly reduces the effectiveness of recognition. Main feature of the proposed adaptive approach consists in the elimination of arbitrariness in the choice of the parameter set. Therefore, the adaptive method is less sensitive to such factors as, e.g., researchers experience, peculiarities of the analyzed data, etc.

From physiological point of view, the obtained results demonstrate that $\mathrm{IH}$ in newborn rats is characterized by essential changes in CVBF. Indeed, rats with $\mathrm{IH}$ show significant dilations of the superior sagittal vein with a reduced velocity of blood flow reflecting a decrease of the cerebral venous blood outflow and the development of venous insufficiency. The latter is in accordance with clinical data in newborns..$^{3,5}$ Other studies also concluded that the suppressed CVBF closely correlates with IH in newborns..$^{9,33-35}$

A crucial role in regulation of cerebral hemodynamics is associated with the sympathetic nervous system. ${ }^{6,7}$ It was shown that the adrenergic vasoconstriction of cerebral vessels represents a protective mechanism for the brain during $\mathrm{IH}^{8-12}$ To study adrenergic influences on CVBF we analyzed reactivity of the sagittal vein to adrenaline in healthy rats and in rats with IH. We found that effects of adrenaline depend on physiological conditions of newborn rats. Under normal state, adrenaline induces constrictions of the sagittal vein. Adrenaline is significantly less effective during IH when it does not have essential influences on the tone of cerebral veins. These facts allow us to conclude that are activity of cerebral veins to adrenaline can be one of the possible mechanisms responsible for pathological changes in $\mathrm{CVBF}$.

\section{Conclusion}

In this study, we proposed the adaptive waveletbased approach being able to estimate sensitive markers quantifying reactions of brain vessels to external factors. Unlike the typically used continuous wavelet transform with empirically selected parameters, this adaptive approach enables us to perform an optimal choice of the parameter set aiming to reveal the most essential distinctions between normal and pathological dynamics of CVBF. Using sequences of DOCT images recorded before and after the administration of adrenaline, we studied different responses of the sagittal vein in normal rats and in animals with stress-induced IH. We found a relation between the incidence of $\mathrm{IH}$ in newborn rats and a suppression of CVBF with the development of venous insufficiency and the 
areactivity to adrenaline. Aiming to quantify reactions of CVBF, we introduced a threshold value of the objective function $R_{d}$. Fairly weak responses to the administration of adrenaline are revealed in rats with IH. This are activity of the cerebral veins to adrenaline can be treated as a possible mechanism responsible for pathological changes in CVBF. The proposed approach improves diagnostic abilities of standard techniques used for processing DOCT data. It could advance understanding of mechanisms underlying IH in newborns.

\section{Acknowledgments}

This study was supported in part by the Russian Foundation for Basic Research (grants 11-0200560-a, 12-02-31204) and by the RF Ministry of Education and Sciences within the Federal program "Scientific and scientific-pedagogic staff of innovative Russia for 2009-2013" (contracts 14.B37.21.0216, 14.B37.21.0853).

\section{References}

1. J. Baun, "Neonatal intracranial hemorrhage," $J$. Diagn. Med. Sonogr. 7, 121-131 (1991).

2. R. W. Leech, P. Kohnen, "Subependymal and intraventricular hemorrhages in the newborn," $A m$. J. Pathol. 77, 465-475 (1974).

3. B. C. Lee, A. E. Grassi, A. Schechner, "Neonatal intraventricular hemorrhage: A serial computed tomography study," J. Comput. Assist. Tomogr. 3, 483-490 (1979).

4. P. Ballabh, "Intraventricular hemorrhage in premature infants: Mechanism of disease," Pediatr. Res. 67, 1-8 (2010).

5. P. Ballabh, A. Braun, M. Nedergaard, "Anatomic analysis of blood vessels in germinal matrix, cerebral cortex, and white matter in developing infants," Pediatr. Res. 56, 117-124 (2004).

6. D. W. Busija, D. D. Heistad, "Factors involved in the physiological regulation of cerebral circulation," Rev. Physiol. Biochem. Pharmacol. 101, 161-211 (1984).

7. T. A. McCalden, "Sympathetic control of the cerebral circulation," J. Auton. Pharmacol. 1, 421-431 (1981).

8. P. A. Cassaglia, R. I. Griffiths, A. M. Walker, "Sympathetic nerve activity in the superior cervical ganglia increases in response to imposed increases in arterial pressure," Am. J. Physiol. 294, R1255R1261 (2008).
9. J. L. Di Gennaro, D. C. Mack, A. Malakouti, J. J. Zimmerman, W. Armstead, M. S. Vavilala, "Use and effect of vasopressors after pediatric traumatic brain injury," Dev. Neurosci. 32, 420-430 (2011).

10. P. Sookplung, A. Siriussawakul, A. Malakouti, D. Sharma, J. Wang, M. Souter, R. Chesnut, M. Vavilala, "Vasopressor use and effect on blood pressure after severe adult traumatic brain injury," Neurocrit. Care 15, 46-54 (2011).

11. S. N. Kroppenstedt, U. W. Thomale, M. Griebenow, O. W. Sakowitz, K. D. Schaser, P. S. Mayr, A. W. Unterberg, J. F. Stover, "Effects of early and late intravenous norepinephrine infusion on cerebral perfusion, microcirculation, brain-tissue oxygenation, and edema formation in brain-injured rats," Crit. Care Med. 31, 2211-2221 (2003).

12. D. Pfister, S. P. Strebel, L. A. Steiner, "Effects of catecholamines on cerebral blood vessels in patients with traumatic brain injury," Eur. J. Anaesthesiol. 42, 98-103 (2008).

13. P. Sandor, "Nervous control of the cerebrovascular system: Doubts and facts," Neurochem. Int. 35, 237-259 (1999).

14. J. H. Meek, L. Tyszczuk, C. E. Elweel, J. S. Wyatt, "Low cerebral blood flow is a risk factor for severe intraventricular hemorrhage," Arch. Dis. Child. Fetal Neonatal Ed. 81, F15-F18 (1999).

15. R. J. Thorburn, A. P. Lipscomb, A. L. Stewart, E. O. Reynolds, P. L. Hope, "Timing and antecedents of periventricular haemorrhage and of cerebral atrophy in very preterm infants," Early Human Dev. 7, 221-238 (1982).

16. L. Jordan, A. Hillis, "Hemorrhagic stroke in children," Pediatr. Neurol. 36, 73-80 (2007).

17. L. Vries, F. Groenendaal, "Patterns of neonatal hypoxicischemic brain injury," Neuroradiology 52, 555-566 (2010).

18. L. C. Jordan, J. T. Kleinman, A. E. Hillis, "Intracerebral hemorrhage volume predicts poor neurologic outcome in children," Stroke 40, 16661671 (2009).

19. J. K. Lynch, K. B. Nelson, "Epidemiology of perinatal stroke," Curr. Opin. Pediatr. 13, 499-505 (2003).

20. L. L. Beslow, D. J. Licht, S. E. Smith, P. B. Storm, G. G. Heuer, R. A. Zimmerman, A. M. Feiler, S. E. Kasner, R. N. Ichord, L. C. Jordan, "Predictors of outcome in childhood intracerebral hemorrhage: A prospective consecutive cohort study," Stroke 41, 313-318 (2010).

21. W. D. Lo, "Childhood hemorrhagic stroke: An important but understudied problem," J. Child Neurol. 26, 1174-1185 (2011).

22. D. Huang, E. A. Swanson, C. P. Lin, J. S. Schuman, W. G. Stinson, W. Chang, M. R. Hee, T. Flotte, 
K. Gregory, C. A. Puliafito, J. G. Fujimoto, "Optical coherence tomography," Science 254, 1178-1181 (1991).

23. P. H. Tomlins, R. K. Wang, "Theory, developments and applications of optical coherence tomography," J. Phys. D Appl. Phys. 38, 2519 (2005).

24. W. Drexler, J. G. Fujimoto, Optical Coherence Tomography: Technology and Applications, Springer, New York (2008).

25. S. G. Mallat, A Wavelet Tour of Signal Processing, Academic Press, New York (1998).

26. P. S. Addison, The Illustrated Wavelet Transform Handbook: Introduction Theory and Applications in Science, Engineering, Medicine and Finance, IOP Publishing, Bristol (2002).

27. S. Thurner, M. C. Feurstein, M. C. Teich, "Multiresolution wavelet analysis of heartbeat intervals discriminates healthy patients from those with cardiac pathology," Phys. Rev. Lett. 80, 15441547 (1998).

28. P. Ch. Ivanov, L. A. Nunes Amaral, A. L. Goldberger, S. Havlin, M. G. Rosenblum, Z. R. Struzik, H. E. Stanley, "Multifractality in human heartbeat dynamics," Nature 399, 461-465 (1999).

29. A. N. Pavlov, V. S. Anishchenko, "Multifractal analysis of complex signals," Phys. Usp. 50, 819-834 (2007).
30. O. V. Semyachkina-Glushkovskaya, V. V. Lychagov, O. A. Bibikova, I. A. Semyachkin-Glushkovskiy, S. S. Sindeev, E. M. Zinchenko, M. M. Kassim, H. A. Braun, F. Al-Fatle, L. A. Hassani, V. V. Tuchin, "The assessment of pathological changes in cerebral blood flow in hypertensive rats with stress-induced intracranial hemorrhage using Doppler OCT: Particularities of arterial and venous alterations," $J$. Photon. Laser. Med. 2, 109-116 (2013).

31. J. C. Spall, Introduction to Stochastic Search and Optimization, Wiley, New Jersey (2003).

32. M. C. Fu, "Optimization for simulation: Theory vs. practice," INFORMS J. Comput. 14, 192-227 (2002).

33. H. S. Ghazi-Birry, W. R. Brown, D. M. Moody, V. R. Challa, S. M. Block, D. M. Reboussin, "Human germinal matrix: Venous origin of hemorrhage and vascular characteristics," Am. J. Neuroradiol. 18, 219-229 (1997).

34. B. G. Carter, W. Butt, A. Taylor, "ICP and CPP: Excellent predictors of long-term outcome in severely brain-injured children," Childs Nerv. Syst. 24, 245-251 (2008).

35. A. Català-Temprano, G. C. Teruel, F. J. C. Lasaosa, O. M. Pons, J. A. Noguera, R. A. Palomeque, "Intracranial pressure and cerebral perfusion pressure as risk factors in children with traumatic brain injury," J. Neurosurg. 106, 463-466 (2007). 